\title{
Eulerian multiphase CFD analysis of particle transport and deposition in the human
}

\author{
R.F. Kunz', D.C. Haworth', L.S. Leemhuis', A.C. Davison', \\ S. Zidowitz ${ }^{2}$ \& A. Kriete ${ }^{3}$ \\ 'Applied Research Laboratory, The Pennsylvania State University, \\ University Park, PA, USA \\ ${ }^{2}$ Center for Medical Diagnostic Systems and Visualization, \\ Universitaetsallee 29, Bremen, Germany. \\ ${ }^{3}$ Image Processing Laboratory, Institute of Anatomy and Cell Biology, \\ Aulweg 123, Giessen, Germany
}

\begin{abstract}
The authors' recent research in the application of three-dimensional, multiphase Computational Fluid Dynamics (CFD) analysis of particle transport and deposition in the human lung is summarized in this paper. A physically accurate representation of the trachea and upper bronchi generations are obtained using high-resolution computed tomography (HRCT) of a rubber cast of an adult human tracheobronchial tree. A computer model of this data is used as a bounding surface for a hybrid unstructured CFD mesh. The differential model employed in the analyses is the ensemble averaged n-fluid system, wherein continuity, momentum, and turbulence quantity conservation equations are solved for an arbitrary number of constituents, here one carrier phase (air) and multiple particulate fields representing different characteristic sizes. Models for non-equilibrium interfacial transfer are incorporated to account for particle drag, dispersion, and deposition. In the present work, steady state simulations were performed corresponding to a nominal adult human inhalation rate $770 \mathrm{of} \mathrm{cm}^{3} / \mathrm{s}$. It is found that the detailed geometric and physical modeling employed gives rise to qualitatively and quantitatively correct deposition rate predictions for particle size ranges of pharmaceutical interest.
\end{abstract}




\section{Introduction}

Respiratory delivery of pharmaceuticals can be attractive in that doses can be introduced into the blood stream rapidly and conveniently. For this reason, a good deal of research has been carried out to optimize the efficiency of inhaled delivery systems. Such work involves elements of substance manufacturing, particle/droplet atomization, inhaler design, lung tissue absorption, and fluid dynamics. Assessing the effectiveness of delivery systems involves some form of absorption measurement, but local measurements within the lung are difficult due to the complex geometry of the lung and the inherent difficulties associated with instrumenting a living subject. Accordingly, non-local measurements, such as blood sampling, have historically been utilized to provide comparative performance data. Recently, non-invasive, optical techniques have been developed (see Mobley and Hochhaus [1]). These techniques provide direct or indirect local absorption information, but are evolving in their maturity, and have accuracy and safety limitations.

The inherent difficulties with measuring human respiratory deposition have motivated a range of modeling efforts that span in complexity from one-dimensional models through three-dimensional computational fluid dynamics (CFD) analysis. 1D models are generally empirical in nature, modeling the lung as a multigeneration symmetric tree (see Yu and Diu [2], for example) with statistical models for relevant geometric quantities (e.g., total number, length, diameter, bifurcation angle for each generation) and bulk deposition rate models accommodating convection, sedimentation and dispersion physics. Models of this type have been widely developed and explored, and are the basis of pharmaceutical industry assessment tools. However, these models are empirical in nature and thereby unreliable if applied outside their range of calibration. Also, details of lung morphology, and three-dimensional/secondary flows are not accounted for directly.

In the present work, three-dimensional, multiphase CFD analysis is employed in the analysis of lung deposition. The motivation for adopting this level of modeling is that, in principle, drug deposition can be predicted much more accurately than with $1 \mathrm{D}$ models. This is because one can directly utilize clinically obtained multidimensional geometry, explicitly resolve multidimensional carrier phase fluid mechanics (e.g., convection, diffusion, secondary motions, flow splits) and apply local conditions based physical models for important deposition flow physics associated with the particles (e.g., buoyancy/sedimentation, drag, dispersion, wall deposition). The approach taken here is based on employing an accurate, threedimensional representation of the human lung, obtained from high-resolution computed tomography of a cast of an adult human lung. Attendant to the inherent complexity of the geometry, large hybrid unstructured meshes are constructed to discretize the computational domain. An Eulerian n-fluid differential model is employed, wherein the ensemble averaged continuity and Navier-Stokes equations are solved for a continuous carrier field (air) and multiple particulate fields, each 
characterized by a representative particle size. The dynamics associated with particle transport are accounted for by using models for drag, dispersion and wall deposition that appear as closure terms in the averaged governing differential system. A code based on modern CFD technology (parallel, unstructured, high order, $n$-fluid) is employed in the numerical simulation of the model.

Several groups have employed multidimensional, multiphase CFD techniques in the analysis of respiratory flows. For example, Asgharian and Anjilvel [3] and Zhang et. al. [4] have used an Eulerian-Lagrangian approach (for carrier and particle phases respectively) and applied their code to geometrically simplified bronchial branch elements and obtained qualitatively good results. To the knowledge of the authors, two groups, in addition to the present authors, are currently involved in multiphase CFD analysis of a highly geometrically accurate human lung model (a European consortium, COPHIT [5] and researchers at Pacific Northwest National Laboratory in the US [6]). As in the present work, these efforts pursue the benefits of modern medical imaging and CFD technologies to provide accurate local predictions of particle deposition.

This paper is organized as follows: First, an overview of the surface definition and volume grid generation is provided. Next details of the physical modeling and numerics are summarized. Results obtained to date are then presented, with emphasis placed on local and bulk particle deposition predictions for various particle sizes.

\section{Geometric modeling}

\subsection{Lung surface definition}

The geometric model that was used here is based on a rubber cast of an adult human tracheobronchial tree that also includes the system of vessels of the left lobe. A photo of the complete cast is given in figure. From this rubber cast, a set of digital volume data was produced by using high-resolution computed tomography (HRCT), with a pixel dimension of $0.35 \mathrm{~mm} \mathrm{x} 0.35 \mathrm{~mm}$ and with $0.4 \mathrm{~mm}$ distance between slices. The quality of resolution of this data set goes far beyond what can be obtained in clinical imaging, due to the absence of bones and tissue that limit the contrast, absence of motion artifacts, and the high degree of the radiation doses applicable. The resulting HRCT volume data set was segmented with a thresholdbased algorithm to archive a binary volume representation of the trachea and the connected bronchial tubes, with focus from the trachea to the main stem bronchi. The gray value volume data as well as the binary segmentation result were edited under visual control to overcome minor imperfections of the rubber cast. For image processing, we used the package ILAB 4 (Schröcker et. al. [7]).

Surface extraction based on the binary volume data was established by a marching cube algorithm (Lorensen and Cline [8]). A volume-preserving algorithm was applied to the resulting polygonal surface representation in order to reduce small scale noise artifacts and discretization artifacts in the data. The final surface 


\section{Simulations in Biomedicine $V$}

representation consists of about 81000 triangles. Finally, the surface geometry was exported into a stereolithographic ('.stl') format to allow further processing with commercial grid generation packages. This data set contains distinct anatomical structures like regular imprints of the hyaline $\mathrm{C}$ - shaped cartilage rings around the trachea and the primary bronchi visible in figure 2 , so that a realistic modeling of the gas flow can be achieved.

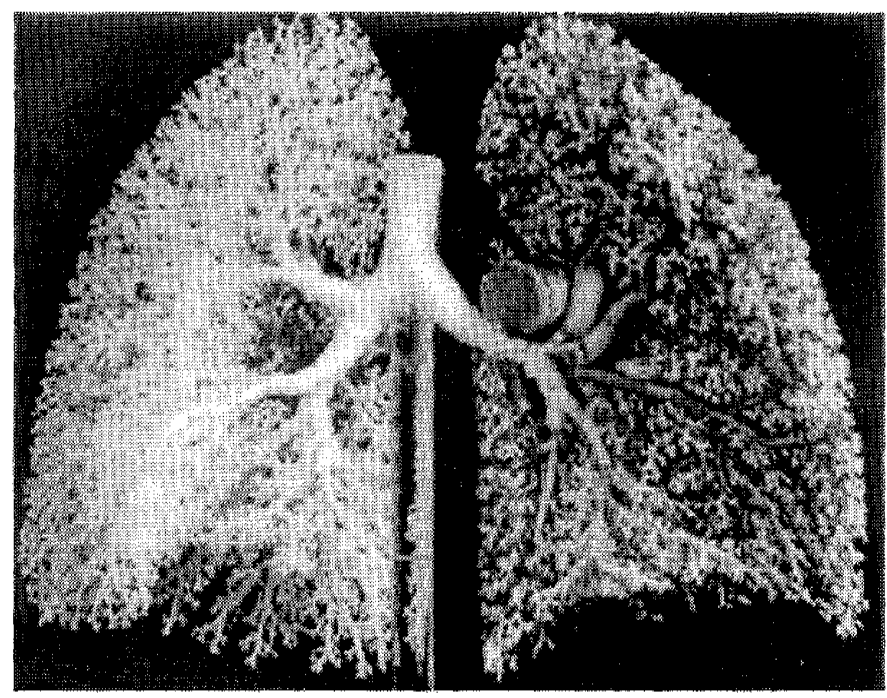

Figure 1: Photograph of the cast model used for surface reconstruction of the first several bronchi generations

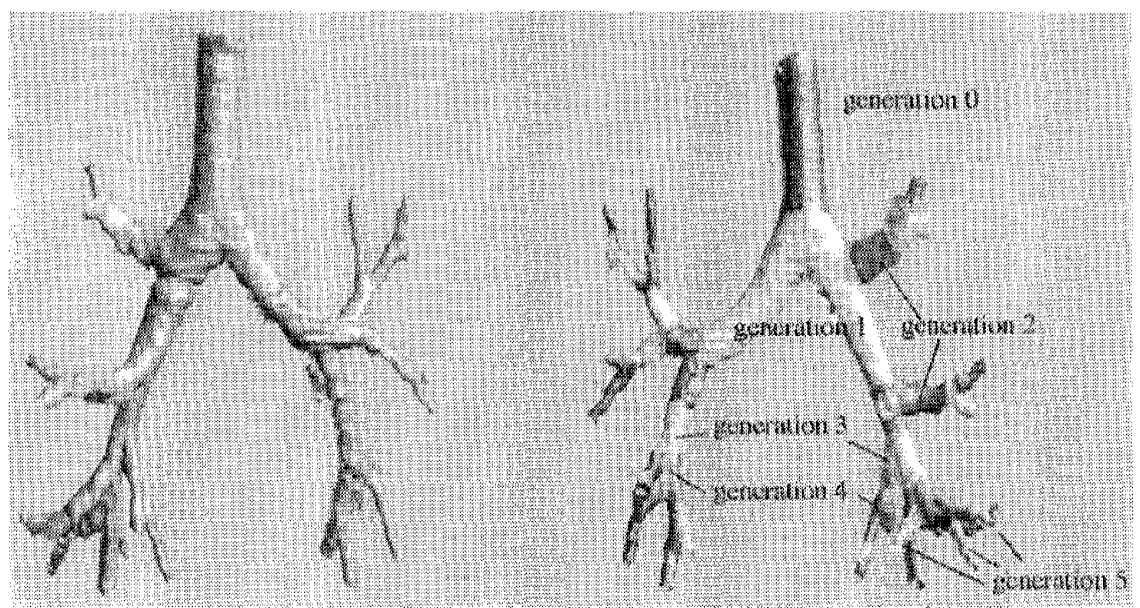

Figure 2a: Surface front view of final .stl model, b) Designation of generations for postprocessing (rear view). 


\subsection{Volume grid generation}

The software package ICEM CFD 4.2 [9] was used to generate the unstructured grid on the lung model. The surface geometry definition described above was imported as an ".stl" file into the software, and the TETRA meshing module was used to generate a triangular surface domain and an interior tetrahedral mesh. In order to accurately represent the detailed geometry in the smaller branches of the model, parameters within TETRA were set such that an adequate number of triangular surface domains existed on each of the terminal bronchi outlets. In this case, the height of the triangles measured approximately $10 \%$ to $15 \%$ of the diameter of the smallest branch. To retain accuracy of the solution throughout the domain of interest, these sizes were kept constant throughout the interior of the volume to be meshed. After the mesh was complete, the iterative smoothing capabilities within ICEM were utilized to increase the quality of the mesh and to maximize the isotropy of the tetrahedral elements.

In order to model near-wall physics appropriately, the PRISM module within ICEM was used to generate a single layer of prism elements along the boundary surfaces of the model. The thickness of this prism layer is approximately half the height of an average tetrahedron. As the tetrahedral elements near the walls were distorted and in some cases replaced by the newly generated prism elements, the quality of the mesh was decreased. After the iterative smoothing and manual editing was completed on the hybrid mesh, the quality was slightly lower than in the original mesh, but still was deemed high enough to yield an accurate solution. The final mesh contained a total of 456,463 prism and tetrahedral elements.

Once the mesh was complete, the inlet of the trachea, each of the 29 terminal bronchi outlet boundaries, and the walls of each successive branch generation were labeled. These family labels were necessary in order to apply the inlet and outflow boundary conditions. Also, the wall labels were useful during the post-processing in order to determine the volume fraction of particles deposited at each level of branching.

\section{Theoretical formulation}

\subsection{Differential model}

An ensemble averaged, single-pressure, $n$-fluid differential system is employed. The continuity and momentum equations, cast in Cartesian coordinates, are presented in eqn (1).

$$
\begin{gathered}
\frac{\partial}{\partial \mathrm{x}_{\mathrm{j}}}\left(\alpha^{k} \rho^{k} \mathrm{u}_{\mathrm{j}}^{k}\right)=-\Gamma^{k} \\
\frac{\partial}{\partial \mathrm{x}_{\mathrm{j}}}\left(\alpha^{k} \rho^{k} \mathrm{u}_{\mathrm{j}}^{k} \mathrm{u}_{\mathrm{i}}^{k}\right)=-\alpha^{k} \frac{\partial \mathrm{p}}{\partial \mathrm{x}_{\mathrm{i}}}+\alpha^{k} \rho^{k} \mathrm{~g}_{\mathrm{i}}+\frac{\partial}{\partial \mathrm{x}_{\mathrm{j}}}\left(\alpha^{k} \mu_{\mathrm{t}}^{k} \frac{\partial \mathrm{u}_{\mathrm{i}}^{k}}{\partial \mathrm{x}_{\mathrm{j}}}\right)+\mathrm{M}_{\mathrm{i}}^{l k}+\sum_{k \neq l} \mathrm{D}^{k l}\left(\mathrm{u}_{\mathrm{i}}^{l}-\mathrm{u}_{\mathrm{i}}^{k}\right)-\Gamma^{k} \mathrm{u}_{\mathrm{i}}^{k}
\end{gathered}
$$


Here, superscript $k$ is the constituent or "field" designator. In the present work, we consider adiabatic flows and each field's density is taken as constant. Interfacial drag and non-drag forces, $\mathrm{D}^{k l}$ and $\mathrm{M}_{\mathrm{i}}^{l k}$, respectively, appear in the momentum equation. In these force terms, superscripts $k$ and $l$ represent donor and receptor fields. The term $-\Gamma^{\mathrm{k}}$ represents a modeled deposition mass flux sink which is non-zero for particle fields.

\subsection{Physical Modeling}

An eleven-field prescription is employed here. The carrier air is represented by field 1 and fields $2-11$ correspond to particle fields of characteristic particle diameters $.0625, .125, .25, .5,1,2,4,8,16$ and $32 \mu \mathrm{m}$ respectively. To account for turbulence in the carrier field, a high-Reynolds number $k-\varepsilon$ turbulence model (Jones and Launder [10]) is employed, i.e., for gas field $k=1$ :

$$
\begin{aligned}
& \frac{\partial}{\partial \mathrm{x}_{\mathrm{j}}} \alpha^{k} \rho^{k} \mathrm{u}_{\mathrm{j}}^{k} \mathrm{k}^{k}=\frac{\partial}{\partial \mathrm{x}_{\mathrm{j}}}\left(\alpha^{k} \frac{\mu_{\mathrm{t}}^{k}}{\mathrm{P}_{\mathrm{rk}}^{k}} \frac{\partial \mathrm{k}^{k}}{\partial \mathrm{x}_{\mathrm{j}}}\right)+\mathrm{P}_{\mathrm{k}}^{k}-\alpha^{k} \rho^{k} \varepsilon^{k}-\Gamma^{k} \mathrm{k}^{k} \\
& \frac{\partial}{\partial \mathrm{x}_{\mathrm{j}}} \alpha^{k} \rho^{k} \mathrm{u}_{\mathrm{j}}^{k} \varepsilon^{k}=\frac{\partial}{\partial \mathrm{x}_{\mathrm{j}}}\left(\alpha^{k} \frac{\mu_{\mathrm{t}}^{k}}{\mathrm{P}_{\mathrm{r} \varepsilon}^{k}} \frac{\partial \varepsilon^{k}}{\partial \mathrm{x}_{\mathrm{j}}}\right)+\mathrm{C}_{1} \frac{\varepsilon^{k}}{\mathrm{k}^{k}} \mathrm{P}_{\mathrm{k}}^{k}-\mathrm{C}_{2} \frac{\varepsilon^{k}}{\mathrm{k}^{k}} \alpha^{k} \rho^{k} \varepsilon^{k}-\Gamma^{k} \varepsilon^{k} \\
& \mu_{\mathrm{t}}^{k}=\mathrm{C}_{\mu} \rho^{k} \mathrm{k}^{k^{3 / 2}} / \varepsilon^{k}
\end{aligned}
$$

Standard high-Reynolds number wall functions are used to account for wall-shear. This gives rise to wall grid spacing requirements, nominally $30<\mathrm{y}^{+}<300$, that are accommodated in the grid generation process.

We employ a standard drag model based on an empirically derived fit to data available for spherical particles in an incompressible flow (Loth [11]):

$$
\begin{aligned}
& \mathrm{D}^{g p}=\frac{3}{4 \mathrm{~d}_{\mathrm{p}}} \mathrm{C} \rho^{g}\left|\mathrm{u}_{\mathrm{i}}^{g}-\mathrm{u}_{\mathrm{i}}^{p}\right| \alpha^{p}, \mathrm{C}_{\mathrm{D}}=\frac{24}{\operatorname{Re}_{\mathrm{p}}} \mathrm{f}, \operatorname{Re}_{\mathrm{p}} \equiv \frac{\rho^{g}\left|\mathrm{u}_{\mathrm{i}}^{g}-\mathrm{u}_{\mathrm{i}}^{p}\right| \mathrm{d}_{\mathrm{p}}}{\mu^{\mathrm{g}}} \\
& \left\{\begin{array}{l}
\mathrm{f}=1+0.1875 \operatorname{Re}_{\mathrm{p}}, \operatorname{Re}_{\mathrm{p}}<1 \\
\mathrm{f}=1+0.1935 \operatorname{Re}_{\mathrm{p}}{ }^{0.6305}, 1<\operatorname{Re}_{\mathrm{p}}<285 \\
\mathrm{f}=1+0.015 \operatorname{Re}_{\mathrm{p}}+0.2283 \operatorname{Re}_{\mathrm{p}}{ }^{0.427}, 285<\operatorname{Re}_{\mathrm{p}}<2000 \\
\mathrm{f}=0.44 \operatorname{Re}_{\mathrm{p}} / 24
\end{array}\right\}
\end{aligned}
$$

The effect of turbulence on the particle fields is accounted for following Aquino and Drew [12], and Carrica et. al. [13]. Specifically, we assume that for these dispersed flows, gas field turbulence drives the particle turbulence. Accordingly, the effective diffusion term for the particle fields is replaced with [12]:

$$
\frac{\partial}{\partial \mathrm{x}_{\mathrm{j}}}\left(\alpha^{p} \mu_{\mathrm{t}}^{p} \frac{\partial \mathrm{u}_{\mathrm{i}}^{p}}{\partial \mathrm{x}_{\mathrm{j}}}\right) \rightarrow \frac{\partial}{\partial \mathrm{x}_{\mathrm{j}}}\left(\alpha^{p}\left[\frac{\rho^{p}}{\rho^{g}}\right] \mu_{\mathrm{t}}^{g} \frac{\partial \mathrm{u}_{\mathrm{i}}^{g}}{\partial \mathrm{x}_{\mathrm{j}}}\right)
$$


In addition to the particle turbulence diffusion model given in equation (4), a turbulence dispersion force due to Carrica et. al. [13] is employed:

$$
\mathrm{M}_{\mathrm{i}}^{g p}=\mathrm{D}^{g p} \frac{v_{\mathrm{t}}^{g}}{S \mathrm{c}_{\mathrm{t}}} \frac{\partial \alpha^{p}}{\partial \mathrm{x}_{\mathrm{i}}}
$$

This dispersion model is the only non-drag force employed in the formulation.

In the literature, it is generally accepted that there are three dominant physical mechanisms that contribute to lung aerosol deposition: impaction sedimentation and diffusion (see Yu and Diu [2], for example). At the level of modeling employed here, impaction and sedimentation arise from transport associated with exact convection and buoyancy terms that arise in the momentum equations. Accordingly, a convection-based wall deposition model that accounts for sedimentation and impaction is used:

$$
\Gamma_{\mathrm{c}}^{k}=\int \rho^{k} \alpha^{k} \mathrm{u}_{\mathrm{i}}^{k} \mathrm{dA} \mathrm{A}_{\mathrm{i}, \text { wall }}, \quad k>1
$$

Here, values of particle volume fraction and velocity are taken at the center of the cell adjacent to the wall, and the mass flux is formed with the wall face area vector. For diffusion flux, a standard gradient diffusion approximation is employed, consistent with the high-Reynolds number law-of-the-wall based wall functions that are employed for the carrier phase turbulence. Specifically, the wall diffusion flux is modeled as:

$$
\Gamma_{\mathrm{d}}^{k}=\int \mathrm{J}^{k}\left|\mathrm{~d} \mathrm{~A}_{\mathrm{wall}}\right|, \mathrm{J}^{k}=\frac{\rho^{\mathrm{k}} \alpha_{\mathrm{c}}^{\mathrm{k}} \mathrm{u}^{l^{*}}}{S \mathrm{c}_{\mathrm{t}}\left[\mathrm{u}^{I+}+\mathrm{g}\left(\mathrm{Sc_{ \textrm {m } }}, S \mathrm{c}_{\mathrm{t}}\right)\right]}, \mathrm{k}>1, \mathrm{~g} \equiv 9\left(\frac{S \mathrm{c}_{\mathrm{m}}}{\mathrm{Sc_{ \textrm {t } }}}-1\right)\left(\frac{\mathrm{Sc_{ \textrm {t } }}}{\mathrm{Sc_{ \textrm {m } }}}\right)^{\frac{1}{4}}
$$

The quantities $S c_{m}$ and $S c_{t}$ refer to the molecular and turbulent Schmidt numbers respectively. Relatively little diffusion occurs due to the large particle sizes, and therefore, a molecular Schmidt number is taken as $S c_{m}=1000$. $S c_{t}$ is set equal to 1.0. The function $\mathrm{g}\left(\mathrm{Sc}_{\mathrm{m}}, \mathrm{Sc}_{\mathrm{t}}\right)$ is a form that is used to model turbulent heat and species transport at walls based on a Reynolds analogy. The total deposition mass flux sink in eqns (1) and (2) is taken as $\Gamma^{k}=\Gamma_{\mathrm{c}}^{k}+\Gamma_{\mathrm{d}}^{k}$.

\subsection{Numerical Method}

\subsubsection{Discretization}

The governing equations are discretized using a face-based, cell centered, finite volume method applied to arbitrary polyhedral cell types. For inviscid flux evaluation, a second order upwind scheme is utilized for all scalars except $\mathrm{k}$ and $\varepsilon$, for which a first order upwind scheme is employed. Viscous terms are evaluated using second order accurate central differences. Gradients appearing in pressure gradient and force source terms are also evaluated using second order central differencing. 


\subsubsection{Solution Procedure}

A lagged-coefficient linearization is employed for non-linear convection terms. The discretized governing equation for transport scalar, $\phi^{k}$, is written in $\Delta$-form as:

$$
\begin{aligned}
& {\left[\mathrm{A}_{\mathrm{P}}^{k}+\sum_{k \neq l} \mathrm{D}^{k l}+\frac{\rho^{k} \alpha^{k} \mathrm{~V}}{\Delta \tau}\right] \Delta \phi_{\mathrm{P}}^{k}-\sum_{k \neq l} \mathrm{D}^{l k} \Delta \phi_{\mathrm{P}}^{l}-\sum_{\mathrm{nb}} \mathrm{A}_{\mathrm{nb}}^{k} \Delta \phi_{\mathrm{nb}}^{k}=} \\
& {\left[\sum \mathrm{A}_{\mathrm{nb}}^{k}\left(\phi_{\mathrm{nb}}^{k}\right)^{\mathrm{m}}-\left(\mathrm{A}_{\mathrm{P}}^{k}+\sum_{k \neq l} \mathrm{D}^{k l}\right)\left(\phi_{\mathrm{P}}^{\mathrm{k}}\right)^{\mathrm{m}}-\sum_{k \neq l} \mathrm{D}^{l k}\left(\phi_{\mathrm{P}}^{l}\right)^{\mathrm{m}}\right]+\mathrm{s}^{k}}
\end{aligned}
$$

where $A_{\mathrm{p}}^{k}$ and $A_{\mathrm{nb}}^{k}$ represent cell and neighbor influence coefficients arising from convection, diffusion and implicitly treated source terms, $S^{k}$ corresponds to explicitly treated source terms, and $\Delta \phi^{k} \equiv\left(\phi^{k}\right)^{\mathrm{m}}-\left(\phi^{k}\right)^{\mathrm{m}}$. A local timestepping procedure is employed wherein at each pseudo-timestep, the momentum equations are solved first (for all fields and coordinate directions simultaneously), followed by solution of a mixture mass continuity equation which is constructed based on the SIMPLE-C algorithm. The volume fraction and continuous field turbulence equations are then solved in succession. A standard under-relaxation procedure is employed where an appropriate under-relaxation factor, $\omega$ is selected $(0.3 \leq \omega \leq 0.7)$ and the pseudo-timestep is evaluated from:

$$
\Delta \tau \equiv \omega \rho^{k} \alpha^{k} V I\left[(1-\omega)\left(A_{\mathrm{P}}^{k}+\sum_{k \neq l} \mathrm{~b}^{k l}\right)\right]
$$

Further details on the numerics can be found in Kunz et. al. [14].

\section{Results}

A steady state simulation was performed as an approximation to a deep steady inhalation. An inlet air volumetric flow rate of $773 \mathrm{~cm}^{3} / \mathrm{s}$ was specified as nominally representative of an adult human (Rudolf et. al. [15]). A constant velocity of $2.0 \mathrm{~m} / \mathrm{s}$ was specified normal to the trachea inlet boundary corresponding to this volume flow rate. A turbulence intensity of $10 \%$ and a turbulence length scale of $\cong 15 \%$ of the inlet diameter were specified and used to construct constant inflow values of $k$ and $\varepsilon$ for the continuous field. Particle field inlet velocities were also set to $2.0 \mathrm{~m} / \mathrm{s}$. For the lightly mass loaded particulate flows of interest here, the specified inlet volume fractions of particle fields does not significantly affect the dynamics of the flow, so long as they are small. Accordingly, for the present computations, a volume fraction of $\alpha_{\mathrm{in}, \mathrm{p}}=0.0001$ was specified for all particulate fields, and $\alpha_{\mathrm{in}, \mathrm{g}}=1 .-\Sigma \alpha_{\mathrm{in}, \mathrm{p}}$. The density of the air and particles was set to $1.2 \mathrm{~kg} / \mathrm{m}^{3}$ and $1200 \mathrm{~kg} / \mathrm{m}^{3}$ respectively. The pressure was set to $0 \mathrm{~Pa}$ at each of the 29 terminal bronchi outlet boundaries.

The CFD code was run until the residuals of the non-linear system had all dropped by 3 orders of magnitude or more. The code was instrumented to output wall flux deposition rates along each of the generations designated in figure $2 \mathrm{~b}$. From these absolute values, deposition efficiencies could be computed, based on the inlet mass flux and the cumulative deposition rates to earlier branches. 


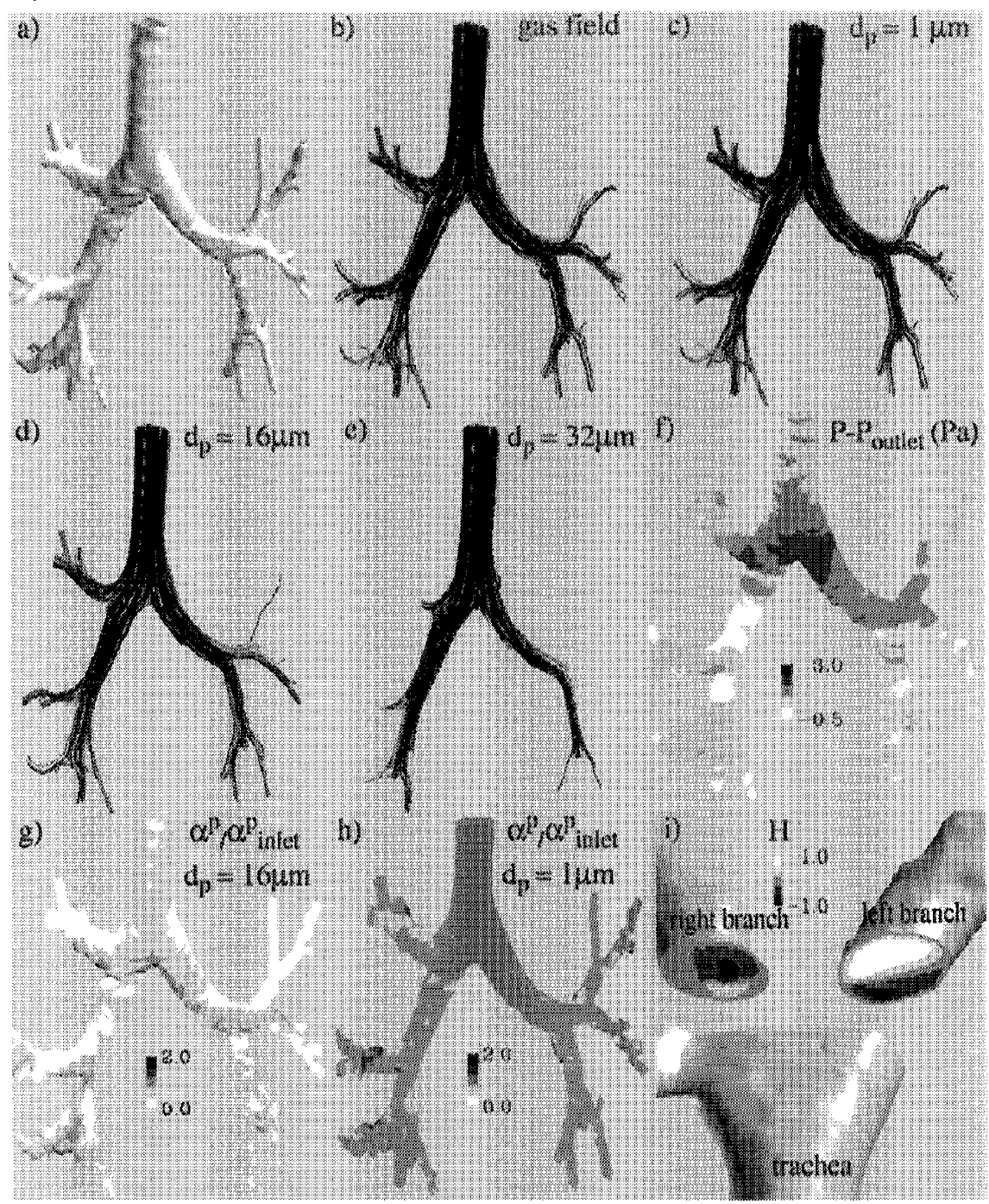

Figure 3 Elements of lung particle deposition simulation. a) Front view of shaded solid model, b) Carrier gas field streamlines seeded from inlet plane, c)-e) Particle field streamlines for $d_{p}=1,16$ and $32 \mu \mathrm{m}$ particles, seeded at same locations as gas field streamlines, $f$ ) predicted surface pressure contours, g)-h) Surface particle field volume fractions $\left(\alpha / \alpha_{\text {inlet }}\right)$ for $d_{p}=16$, and $1 \mu \mathrm{m}$ fields, i) Contours of helicity density at a cut plane just below the trachea-generation 1 branch, illustrating the counterrotating secondary flows in the bends. White indicates a clockwise secondary motion, while black indicates counter-clockwise. 
Figure 3 shows representative results of the simulation. A front view of the lung is provided in figure $3 \mathrm{a}$ for reference. Computed streamlines for the air, $1 \mu \mathrm{m}, 16 \mu \mathrm{m}$ and $32 \mu \mathrm{m}$ particle fields are shown in figures $3 \mathrm{~b}-\mathrm{e}$. It is observed that the $1 \mu \mathrm{m}$ particle streamlines are virtually identical to the gas field, due to the low Stokes number for this particle size $\left(\mathrm{St} \equiv \tau_{\mathrm{p}} / \tau_{\text {mean }}=\left(\rho^{p} \mathrm{~d}_{\mathrm{p}}^{2} / 18 \mu^{\mathrm{g}}\right) /\left(\mathrm{D}_{\text {mean }} / \mathrm{V}_{\text {mean }}\right) \approx \mathrm{O}\left(10^{-4}\right)\right.$, where $D_{\text {mean }}$ and $V_{\text {mean }}$ are nominal mean flow length and velocity scales). The 16 and $32 \mu \mathrm{m}$ particles show successively increased slip between particle and gas fields, with $32 \mu \mathrm{m}$ particle streamlines clearly intersecting the lung walls due to impaction and sedimentation. Figure 3(f) shows surface pressure contours along the lung wall boundaries (note that modified pressure $\mathrm{P}=\mathrm{p}+\rho^{f} \mathrm{gz}$ is plotted, which effectively removes the gravity head from the contours). A richly varying pressure field is observed associated with curvature and blockage induced accelerations and decelerations within the bronchi passages. Figures $3(\mathrm{~g})$ and $(\mathrm{h})$ show predicted particle volume fractions for $16 \mu \mathrm{m}$ and $1 \mu \mathrm{m}$ particles, along the lung wall boundaries. The smaller particles $(1 \mu \mathrm{m})$ exhibit little deviation from the inlet values since very little slip arises in these fields. Several interesting observations can be made from the $16 \mu \mathrm{m}$ result. Most notable is the accumulation of particles due to impaction and sedimentation at branch concave curvatures and at bronchi sections that are facing upward (against gravity). "Starvation" of particle volume fraction is predicted in higher generation branches, especially along convex curvatures and at downward directed lung wall faces. Also observed is a slight reduction in particle volume fraction near the inlet (i.e. $\alpha^{p}<\alpha^{p}{ }_{\text {inlet }}$ ) due to gravity-induced acceleration of the particles from the no-slip relative velocity specified at the inlet. Figure 3(i) illustrates the presence of secondary flows. There, contours of helicity density $\left(H \equiv u_{i} \omega_{i} /\left|u_{i}\right|\left|\omega_{i}\right|\right)$ are plotted. This normalized dot product of the velocity and vorticity vectors is a scalar that can be conveniently used to identify vortical structures in complex geometries. Also, in figure 3(i) the counter-rotating secondary motions associated with turning of the developing trachea boundary layers are easily identified.
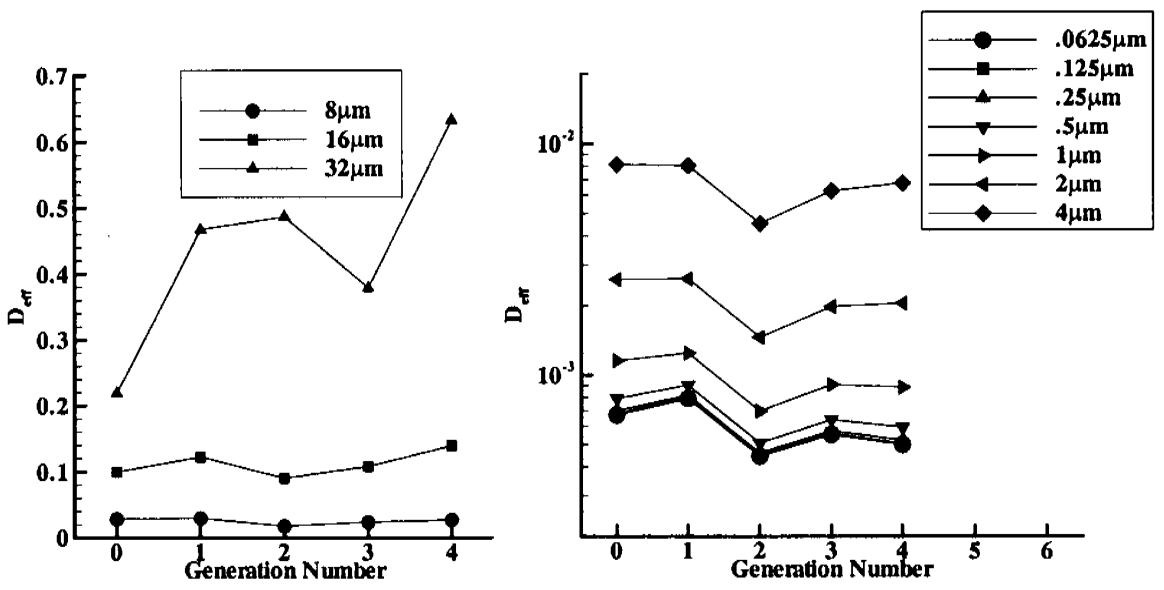

Figure 4 Cumulative deposition rates vs. generation number for the range of particle diameters considered. 
Figure 4 shows cumulative deposition rates vs. generation number for the range of particle diameters considered. Cumulative deposition rate is defined as the particles deposited in the current generation as a percentage of that available from the previous generation. Larger particles show a significant increase in deposition rate once they pass the nearly vertical thorax due to impaction and sedimentation, consistent with figures 3(d), 3(e) and 3(g). The significantly lower deposition rates associated with the smaller particles exhibit relatively flat deposition efficiency profiles with generation as observed in figure 4(b).

In figure 5, total deposition for the entire model is plotted vs. particle diameter. There the convection and diffusion components of the deposition rates are plotted along with total deposition. Convection-induced deposition (i.e., impaction and sedimentation) dominates for particle diameters greater than $1 \mu \mathrm{m}$, approaching $\mathrm{D}_{\mathrm{eff}}=1$ (all particles deposited) for $32 \mu \mathrm{m}$. Diffusion dominated deposition rates are relatively independent of particle diameter below $1 \mu \mathrm{m}$ and drop off significantly at higher particle diameters.

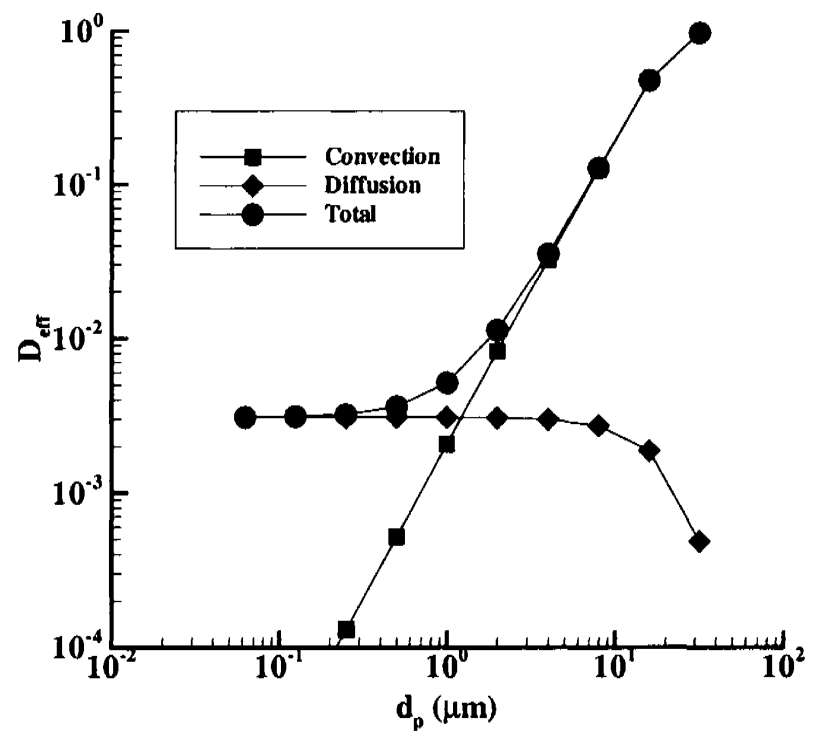

Figure 5 Convection, diffusion and total deposition efficiency predictions for first five generations of tracheobrochial tree for the range of particle diameters considered.

\section{Conclusions}

A three-dimensional multiphase CFD method has been presented and applied for modeling particle deposition in the human lung. In the authors' view, the accuracy inherent in the geometric modeling employed, coupled with unstructured multiphase CFD analysis and physical modeling, provides a modern framework for building an accurate tool for pharmaceutical delivery assessments.

The modeling framework presented is being evolved beyond the baseline capability demonstrated here. Specifically, the basic approach outlined is currently, or will be 
in the future, extended in a number of ways. These advancements include incorporation of improved drag and non-drag interfacial dynamics models, improved deposition models, unsteady boundary conditions to accommodate complete breathing cycles, variable geometry/moving meshes to account for geometric variations in the breathing cycle, incorporation of the upper-trachea, mouth and nasal airway passages, and the representation/modeling of higher generations (i.e., $>5$ considered here).

\section{Acknowledgements}

The authors thank P. Gehr from the Institut of Anatomy at the University of Bern and H. Schulz from the GSF - National Research Center for Environment and Health (Germany) for the provision of the rubber cast of the lung and the tomographic data respectively. This work was supported in part by DFG project \# KR 1921/2-1. The third author is supported under a Penn State Applied Research Laboratory Educational and Foundational fellowship.

\section{Nomenclature}

\subsection{Symbols}

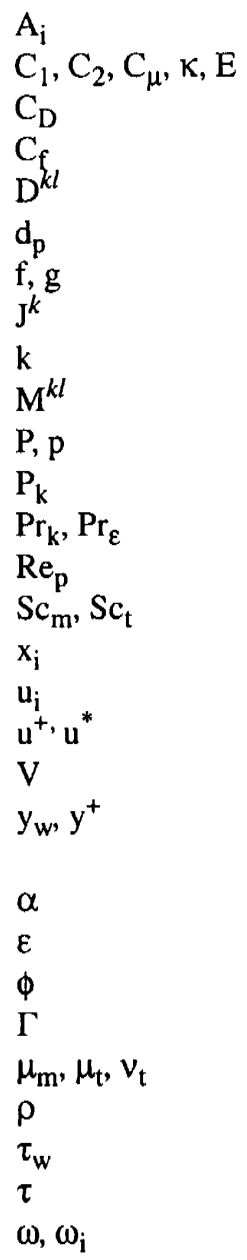

area vector

turbulence model constants

drag coefficient

cell face mass flux

drag force kernel

particle diameter

drag force and deposition modeling functions

diffusive mass flow rate

turbulent kinetic energy

non-drag force

modified and static pressure

turbulence production

turbulent Prandtl numbers

particle Reynolds number

molecular and turbulent Schmidt numbers

Cartesian coordinates

Cartesian velocity components

velocity in inner variables, friction velocity

cell volume

wall proximity in physical, inner variables

volume fraction

turbulent dissipation rate

general transport scalar

deposition mass flux

molecular, eddy viscosity, kinematic eddy viscosity

density

wall shear stress

pseudo-timestep

under-relaxation factor, vorticity vector 


\subsection{Superscripts and subscripts}

$\begin{array}{ll}\mathrm{c}, \mathrm{d} & \text { convection, diffusion } \\ \mathrm{g}, \mathrm{p} & \text { gas, particle field } \\ \mathrm{k}, \mathrm{l} & \text { field indicators } \\ \mathrm{m} & \text { pseudo-timestep indicator } \\ \mathrm{P}, \mathrm{nb} & \text { current, neighbor cell indicators }\end{array}$

\section{References}

[1] Mobley, C., Hochhaus, G., Methods used to assess pulmonary deposition and absorption of drugs. Drug Delivery Today, 6(7), pp. 367-375, April 2001.

[2] Yu, C.P., Diu, C.K., A comparative study of aerosol deposition in different lung models.American Industrial Hygiene Association Journal, 43(1), pp. 54-65, 1982.

[3] Asgharian, B., Anjilvel, S., Inertial and gravitational deposition of particles in a square cross-section bifurcating airway. Aerosol Science and Technology, 20, pp. 177-193, 1994.

[4] Zhang, L., Asgharian, B., Anjilvel, S., Inertial deposition of particles in the human upper airway bifurcations. Aerosol Science and Technology, 26, pp. 97-110, 1997.

[5] http://www.cophit.co.uk

[6] http:/gallery.pnl.gov/mscf/ImageLib.nsf/(\$All)/4ZPTCP?opendocument

[7] F. Schröcker, W. Berghorn, H. Bettag, A. Bohne, J. Breitenborn, H. Hahn, B. Preim, R. Rascher-Friesenhausen, A. Schenk, D. Selle, W. Spindler, H. Jürgens, M. Lang, H.-O. Peitgen, Highly integrated user-friendly research and development platform for medical image processing and visualization. Radiology Suppl., 217, pp. 699, 2001.

[8] Lorensen, W.E. and Cline, H.E., Marching cubes: a high resolution 3D surface reconstruction algorithm. Computer Graphics, 21(4), pp. 163-169, 1987.

[9] ICEM CFD Software User Manual v4.1, ICEM CFD Engineering, Berkeley, CA, 2000.

[10] Jones, W.P., Launder, B.E., The prediction of laminarization with a two-equation model of turbulence. International Journal of Heat and Mass Transfer, 15, pp. 301 $314,1972$.

[11] Loth, E., Numerical approaches for motion of dispersed particles, droplets and bubbles. Progress in Energy and Combustion Science, 26, pp. 161-223, 2000.

[12] Aquino, L.J., Drew, D, Momentum based model for sediment transport. Proc. of the 15th ASCE Engineering Mechanics Conference, 2002.

[13] Carrica, P.M., Drew, D., Bonetto, F., Lahey, R.T., A polydisperse model for bubbly two-phase flow around a surface ship. International Journal of Multiphase Flow, 25, pp. 257-305, 1999.

[14] Kunz, R.F., Yu, W.S., Antal, S.P., Ettorre, S.M. An unstructured two-fluid method based on the coupled phasic exchange algorithm. AIAA Paper 2001-2672, 2001.

[15] Rudolf, G, Kobrich, R., Stahlhofen, Modeling and algebraic formulation of regional aerosol deposition in man. Journal of Aerosol Science, 21(1), pp. S403-S406, 1990. 
